\title{
Prolonged exposure to neutrophil extracellular traps can induce mitochondrial damage in macrophages and dendritic cells
}

\author{
Luis Donis-Maturano', Luvia E Sánchez-Torres², Arturo Cerbulo-Vázquez³, Rommel Chacón-Salinas², \\ Gina S García-Romo4, Mariana C Orozco-Uribe', Juan C Yam-Puc', Marco A González-Jiménez³, \\ Yuriria L Paredes-Vivas ${ }^{3}$, Juana Calderón-Amador', Sergio Estrada-Parra², Iris Estrada-García ${ }^{2}$ \\ and Leopoldo Flores-Romo ${ }^{1 *}$
}

\begin{abstract}
Neutrophils are one the earliest, crucial innate defenses against innumerable pathogens. Their main microbicidal activities include phagocytosis and degranulation, with many pharmacologically active molecules contributing to inflammation. Recently, a novel antimicrobial mechanism was discovered; the Neutrophil Extracelullar Traps (NETs) formed by extrusion of DNA and associated molecules (histones, elastase, antimicrobial peptides, among others) which trap and kill microorganisms. Since NETs were recently described, research has focused on their induction and microbicidal properties, and recently on disease involvement. However, the functional consequences of NETs interacting with other immune cells, either resident or recruited during early inflammation, have not been assessed. We therefore investigated the consequences of exposing two major APCs, macrophages (Mfs) and conventional Dendritic Cells (cDCs) to NETs. Our data revealed that at early times (30 min), both Antigen Presenting Cells (APCs) showed induction of important costimulatory molecules (CD80, CD86). Unexpectedly, however, at later times (6 and 24 hours) NETs apparently triggered a cell death process in these APCs by a caspase- and Apoptosis induced factor (AIF)-dependent pathway, suggesting mitochondrial damage. By rhodamine-123 labelling we found that in both APCs, relatively prolonged exposure to NETs or their components importantly decreased the mitochondrial membrane potential. Ultrastructural analysis confirmed mitochondrial alterations in both APCs.

Our results would suggest that early in inflammation, NETs can activate the two main APCs (Mfs and CDCs), but as the process continues, NETs can then initiate apoptosis of these cells through mitochondrial harm. Conceivable, this "late" induction of cell death in these two APCs might start limiting an ongoing inflammatory process to control it.
\end{abstract}

Keywords: NETs; Dendritic cells; Macrophages; Mitochondrial damage

\section{Introduction}

Polymorfonuclear leukocytes (PMNs) or neutrophils, one of the first effector cells of the innate immune system against infection are the most abundant circulating leukocytes and were discovered by Paul Ehrlich. Neutrophils exhibit numerous molecules to accomplish their functions such as Fc receptors, complement components, antimicrobial peptides, tumour necrosis factor- $\alpha$, interleukin (IL)-1 $\alpha$, IL-1Ra, IL-12, vascular endothelial growth factor,

\footnotetext{
* Correspondence: lefloresromo@gmail.com

'Department of Cell Biology, Cinvestav-IPN. AV. IPN No 2508, Zacatenco C.P. 07330, D.F, México

Full list of author information is available at the end of the article
}

IL-8, growth-related gene product, macrophage inflammatory protein (MIP)- $1 \alpha$, interferon- $\gamma$, among others. PMNs are rapidly recruited to tissues upon injury or infection, where they likely encounter other cells, for instance local and/or recruited dendritic cells and monocytes/macrophages. Until recently neutrophils were thought to perform mainly two essential functions: phagocytosis and degranulation. Upon phagocytosis, PMNs engulf and kill microbes by fusion of phagosomes and lysosomes with granules containing a vast arsenal of antimicrobial molecules (Borregaard and Cowland 1997; Papayannopoulos and Zychlinsky 2009; Segal 2005). During degranulation, neutrophils release to the vicinity of the infectious/inflammatory focus several 
pharmacologically active molecules, some with antimicrobial properties (Borregaard and Cowland 1997; Fujie et al. 1999).

Recently, Brinkman et al., revealed a novel antimicrobial mechanism for PMNs, whereby activated neutrophils release their nuclear DNA and associated molecules (e.g., histones, elastase, LL-37). This mechanism was first described in human PMNs (Brinkmann et al. 2004), and has since been found in mouse (Ermert et al. 2009), bovine (Lippolis et al. 2006), fish (Palic et al. 2007), and cat neutrophils (Wardini et al. 2010), and in chicken heterophils (Chuammitri et al. 2009). Upon activation, these neutrophil extracellular traps (NETs) are released as a result of a cell death process apparently different from apoptosis or necrosis, which was called Netosis. Netosis depends on the generation of reactive oxygen species (ROS) by NADPH oxidase (Fuchs et al. 2007). NETs have been reported in human appendicitis, experimental shigellosis, preeclampsia (Brinkmann et al. 2004), ulcerative colitis (Savchenko et al. 2011), periodontitis (Vitkov et al. 2009), lupus (Garcia-Romo et al. 2011; Lande et al. 2011), smallvessel vasculitis (Kessenbrock et al. 2009), allergy (Dworski et al. 2011), necrotizing fasciitis (Buchanan et al. 2006), pneumococcal pneumonia (Beiter et al. 2006) and malaria (Baker et al. 2008). NETs are induced by a variety of stimuli such as activated platelets, placental micro-debris, LPS, IL-8, TNF- $\alpha$, phorbol 12-myristate 13-acetate (PMA), and by multiple microorganisms including bacteria, fungi and protozoan (Brinkmann et al. 2004; Bruns et al. 2010; Gabriel et al. 2010; von Kockritz-Blickwede and Nizet 2009; Ramos-Kichik et al. 2009).

Most studies about NETs are focused either on their experimental induction or the microbial killing, and few about diseases. However, little attention has been paid to the putative interactions between the many active molecules of NETs and the cellular subsets recruited to the inflammatory site. Here, we have identified previously unrecognized features: that NETs can activate macrophages and conventional dendritic cells, but also that they can cause death later on. Our results suggest that APC damage is at the mitochondrial level and that the cell death process triggered seems caspase- and AIF-dependent. Our data suggest that besides the antimicrobial properties, some molecular components of NETs might display -after some time- a deleterious apoptotic impact upon neighboring cells (including resident and arriving/recruited cells), perhaps to start restraining the ongoing inflammatory reaction.

\section{Materials and methods}

\section{Ethics}

This research was performed on healthy competent volunteers in accordance with the Declaration of Helsinki of the world Medical Association, and the Mexican General Health Law regarding research. The ethics committee of the National School of Biological Sciences approved this study (permission number: "Protocolo \#CEI-ENCB 011/ 2013") and informed written consent was obtained from donors.

\section{Isolation of human neutrophils and NETs formation}

Human blood neutrophils were isolated from healthy donors using Histopaque 1119 and Percoll gradient (Aga et al. 2002). $10^{6}$ healthy neutrophils $/ \mathrm{mL}$ of RPMI-1640 medium supplemented with $2 \%$ fetal bovine serum (FBS) were stimulated with $100 \mathrm{nM}$ Phorbol 12-Myristate 13Acetate (PMA) (SIGMA, cat. P-81-39) at $37^{\circ} \mathrm{C}$ for $4 \mathrm{~h}$ in $5 \% \mathrm{CO}_{2}$ atmosphere to optimally induce NETs. After the period for inducing the NETs, the whole cell suspension was centrifuged at $2500 \mathrm{rpm}$ for $5 \mathrm{~min}$ and the supernatant was carefully collected (NETs supernatant), while the NETs (in the pellet fraction) were washed twice with RPMI-1640 medium supplemented with 2\% FBS to discard the potential effects of -for instance- residual PMA. Thus, this supernatant obtained from the washings of NETs (NETs supernatant) was precisely used as a control (NET sn) to treat the APCs. We checked the NETs sn for the presence of proteins by electrophoresis and by the Bradford method (data not shown). Results shown are the mean $+/$ - Standard Error of Mean of five independent experiments from samples obtained from five different healthy subjects.

\section{NET fluorescence staining}

$10^{6}$ neutrophils/mL of RPMI-1640 medium supplemented with $2 \%$ FBS were stuck on $0.001 \%$ poly-L-lysine-treated glass coverslips (Sigma Aldrich, St. Louis, MO, USA) and incubated $4 \mathrm{~h}$ at $37^{\circ} \mathrm{C}$ in $5 \% \mathrm{CO}_{2}$ atmosphere in Multiwell Plates (Corning Incorparated. Costar ${ }^{\mathrm{R}}$ cat. 3598) with or without stimulus. After incubation, cells were fixed with $4 \%$ paraformaldehyde overnight and blocked $2 \mathrm{~h}$ with $10 \%$ normal mouse serum. Cells were then permeabilized with 0.02\% Triton X-100 (Polysciences Inc. cat. 4605) in $1 \mathrm{M}$ $\mathrm{NaCl}$ and incubated with primary antibodies (mouse antihuman elastase or mouse anti-human histone, both kindly donated by Dr. A. Zychlinsky, Max Planck Institute for Infection Biology, Germany) which were detected with the following secondary antibodies: Alexa Fluor ${ }^{\mathrm{R}} 488$ goat anti-mouse IgG (Molecular Probes, cat. A-11017) and Alexa Fluor ${ }^{\mathrm{R}} 594$ goat anti-mouse IgG (Molecular Probes, cat. A-11020). For DNA detection 4',6-Diamidino-2phenylindole-dihydrochloride (DAPI) was used. Specimens were analyzed with a confocal microscope (Olympus BX51TF, Tokyo, Japan).

\section{Monocyte separation and monocyte-derived macrophages}

Peripheral blood mononuclear cells (PBMC) were isolated from buffy coats of healthy donors by Ficoll-Hypaque 
(Gibco/BRL) density-gradient. CD14 ${ }^{+}$cells were separated by FACS sorting using anti-human CD14-PE (Pharmingen cat. 555398) in the MoFlo ${ }^{\text {Tw }}$ cytometer (Beckman Coulter). The monocytes obtained were on average $97 \%$ pure.

CD14+ monocytes at $10^{6}$ cells $/ \mathrm{ml}$ in 24-well plates (Costar, Cambridge, MA) were cultured in RPMI-1640 plus 5\% human serum, 1 mM HEPES (GIBCO, cat. 15630), $2 \mathrm{mM}$ L-Glutamine (GIBCO, cat. 25030), $100 \mathrm{UI} / \mathrm{mL}$ Penicillin (GIBCO, cat. 15070), 100 $\mu \mathrm{g} / \mathrm{mL}$ Streptomycin (GIBCO, cat. 15070), $50 \mu \mathrm{g} / \mathrm{mL}$ Gentamicin (GIBCO, cat. $15710)$ at $37^{\circ} \mathrm{C}$ in $5 \% \mathrm{CO}_{2}$ atmosphere. Cultures were fed with fresh medium every 2 days for 6 days.

\section{Monocyte-derived dendritic cells}

CD14+ monocytes at $10^{6}$ cells $/ \mathrm{ml}$ in polystyrene 24-well plates (Costar, Cambridge, MA) were cultured in RPMI1640 plus $5 \%$ human serum, 1 mM HEPES (GIBCO, cat. 15630), 2 mM L-Glutamine (GIBCO, cat. 25030), 100 UI/mL Penicillin (GIBCO, cat. 15070), $100 \mu \mathrm{g} / \mathrm{mL}$ Streptomycin (GIBCO, cat. 15070), $50 \mu \mathrm{g} / \mathrm{mL}$ Gentamicin (GIBCO, cat. 15710 ) at $37^{\circ} \mathrm{C}$ in $5 \% \mathrm{CO}_{2}$ atmosphere, supplemented with $1000 \mathrm{U} / \mathrm{ml}$ GM-CSF (PreproTech, cat. 300-03) and $200 \mathrm{ng} / \mathrm{ml} \mathrm{IL-4} \mathrm{(R \& D} \mathrm{System,} \mathrm{cat.}$ 204-IL). Cultures were fed fresh medium and cytokines every 2 days for 6 days.

\section{Stimulation and staining of dendritic cells and macrophages}

To verify monocyte differentiation to macrophages and dendritic cells we used double labeling with CD1a-PE (Santa Cruz Biotechnology, cat. Sc-5265PE) and CD14APC (Pharmingen, cat. 555399) (Caux et al. 1996). CD14 + CD1a- cells were deemed as monocyte-macrophages while CD14-CD1a + cells were considered DCs. These subpopulations were further screened for the expression of HLADR/P/Q (Pharmingen, cat. 3238X), CD80 (Pharmingen, cat. 557227) and CD86 (Pharmingen, cat. 555660).

$10^{6}$ dendritic cells or $10^{6}$ macrophages were stimulated either with NETs (the pellet fraction) obtained from $10^{6}$ stimulated PMNs, NET supernatant (obtained from the washing of $10^{6}$ NETting PMNs) as control, or defined NETs components. Given that we used equal amount of APCs and PMNs to produce the NETs, we describe this as a ratio APCs 1: 1 NETs (we have done also ratios of APCs 1: 2 NETs, shown in Additional file 1: Figure S2). The following NET components were used: Cathepsin G (Sigma Aldrich, Cat. C4428), Elastase (Sigma Aldrich, Cat. E8140), Histone H2A (Sigma Aldrich, Cat. H2042), Myeloperoxidase (Sigma Aldrich, Cat. M6908) and Cathelicidin LL37 (Kindly donated by Dr. L. Cedillo, CINVESTAV, México), during $30 \mathrm{~min}, 3,6,12$ and $24 \mathrm{~h}$. Subsequently, cells were treated $5 \mathrm{~min}$ with universal blocking reagent (Biogenex, cat HK085-5K) and stained with different combinations of purified antibodies: anti- human HLA DR (Alphachain, cat. M0746), HLA DR/P/ Q-FITC (Pharmingen, cat. 32384X), CD80-PE (Pharmingen, cat. 557227) and CD86-APC (Pharmingen, cat. 555660), and Viability Staining Solution: 7-Amino-Actinomycin-D (Pharmingen, cat. 55925).

To evaluate caspase dependent and independent pathways, we used the Fixation/Permeabilization kit (BD cytofix/ cytoperm $^{\text {tw }}$ cat. 554714) and Rabbit anti-active caspase-3 FITC (Pharmingen, cat. 559341), rabbit anti-human AIF (Abcam, cat. Ab32516), Alexa Fluor ${ }^{\mathrm{R}} 488$ goat anti-rabbit IgG (Molecular Probes, cat. A-11008).

To assess the mitochondrial membrane potential, macrophages or dendritic cells previously stimulated with NETs were harvested, washed with PBS and stained with Rhodamine-123 (SIGMA, cat. R8004) for $30 \mathrm{~min}$ at room temperature. After this, cells were washed twice with $2 \mathrm{~mL}$ of PBS $1 \mathrm{X}$ and analyzed by flow cytometry. As a positive control for the induction of AIF and mitochondrial membrane depolarization, we used carbonyl cyanide m-chlorophenyl hydrazone (CCCP) (Lim et al. 2001; de Graaf et al. 2004) and PBS for caspase-3.

\section{Transmission electron microscopy}

Cell suspensions were fixed 90 min with of $2.5 \%$ glutaraldehyde and $4 \%$ paraformaldehyde, postfixed $90 \mathrm{~min}$ with $1 \%$ osmium tetroxide and gradually dehydrated with increasing concentrations of anhydrous ethanol (70, 80, 96 and 100\%) and embedded in Epon and absolute alcohol. After polymerization, specimens were cut at $60 \mathrm{~nm}$ and contrasted with with uranyl acetate and lead citrate and finally examined by transmission electron microscopy (Zeiss EM10).

\section{Statistics}

Statistics were performed with one-way ANOVA using a Tukey and Bonferroni $t$-test for all multiples pairwise comparisons using GraphPad Prism 5 project.

\section{Results}

\section{Induction of neutrophil extracellular traps}

Human neutrophils isolated from healthy donors were stimulated or not with PMA to induce NETs. The basic content of this extracellular material was analyzed as previously described (Brinkmann et al. 2004) and found that contains DNA (blue fluorescence), elastase (red fluorescence) and histone (green fluorescence), thus confirming the presence of NETs (Additional file 2: Figure S1).

\section{NETs can activate macrophages and dendritic cells at early time of exposure}

CD80 (B7-1) and CD86 (B7-2) are crucial costimulatory molecules of antigen presenting cells (APCs), CD86 is constitutively expressed and CD80 is slowly induced and is stable for longer periods than CD86 (Bhatia et al. 
2006) therefore we were intrigued by the seemingly bimodal expression of CD86. To assess if NETs would induce APC activation we exposed Mfs or DCs to NETs or to NET supernatants as controls. Mfs and DCs were identified by combining CD1a and CD14 antibodies as described. Exposure of macrophages to NETs increased the HLA-DR + CD80+ macrophages at 24 h (Figure 1A) while HLA-DR + CD86+ macrophages increased at $30 \mathrm{~min}$, 6 and $24 \mathrm{~h}$ (Figure 1B). For DCs cultured with NETs we observed increased percentages both in the HLA-DR + CD80+ and HLA-DR + CD86+ populations at 24 h poststimulation (Figure 1C,D), but the HLA-DR + CD80+ population appeared earlier (30 minutes) (Figure $1 \mathrm{C})$.

\section{Macrophages and dendritic cells start to die upon prolonged exposure to NETs}

7-AAD, an actinomycin $\mathrm{D}$ analog that binds DNA through GC regions has been used to quantify cell death as the membrane integrity is lost and 7-AAD gains access to DNA (O'Brien and Bolton 1995; Schmid et al. 1992; Schmid et al. 1994). When exposing Mfs or DCs to NETs, we observed increased proportions of dead cells using 7-AAD labelling. This appeared since $6 \mathrm{~h}$ until $24 \mathrm{~h}$ after NETs exposure. We found at $24 \mathrm{~h}$ post incubation with NETs $30.58 \%$ and $30.16 \%$ increase of cell death for macrophages (Figure 2A) and DCs (Figure 2B), respectively.
Mitochondrial damage in macrophages and dendritic cells exposed to NETs

The fluorescent dye rhodamine 123 (Rh123) is used to assess the mitochondrial membrane potential $(\Delta \Psi \mu$. Rh123 stains mitochondria directly, and distributes into the mitochondrial matrix in response to $\Delta \Psi \mathrm{m}$ (Chen et al. 1982; Johnson et al. 1980). When we analyzed Rh123 staining in Mfs and DCs, histograms revealed three clearly distinguished peaks where: R1 corresponds to cells without damage (normal $\Delta \Psi \mathrm{m}$ ) in mitochondrial membranes, $\mathrm{R} 2$ represents cells with some damage or medium $\Delta \Psi \mathrm{m}$ and R3 corresponds to severe damage in mitochondrial membranes or low $\Delta \Psi \mathrm{m}$ (Figure $3 \mathrm{~A}$ ). We observed that when adding NETs to macrophages, the percentage of live cells (R1) decreased from 30 min (Figure 3B), while that of cells with mitochondrial damage (R2 and R3) increased (Figure 3C,D). The same happened for DCs exposed to NETs (Figure 3E-G).

Mfs and DCs undergo a caspase- and AIF-dependent cell death after prolonged incubation with NETs

To investigate whether apoptosis was involved in the death of macrophages and DCs, both populations were cultured with NETs from human blood neutrophils. Because we observed changes in the mitochondrial membrane with Rho123 staining, we decided to evaluate active caspase- 3 and AIF, the latter is a molecule involved in
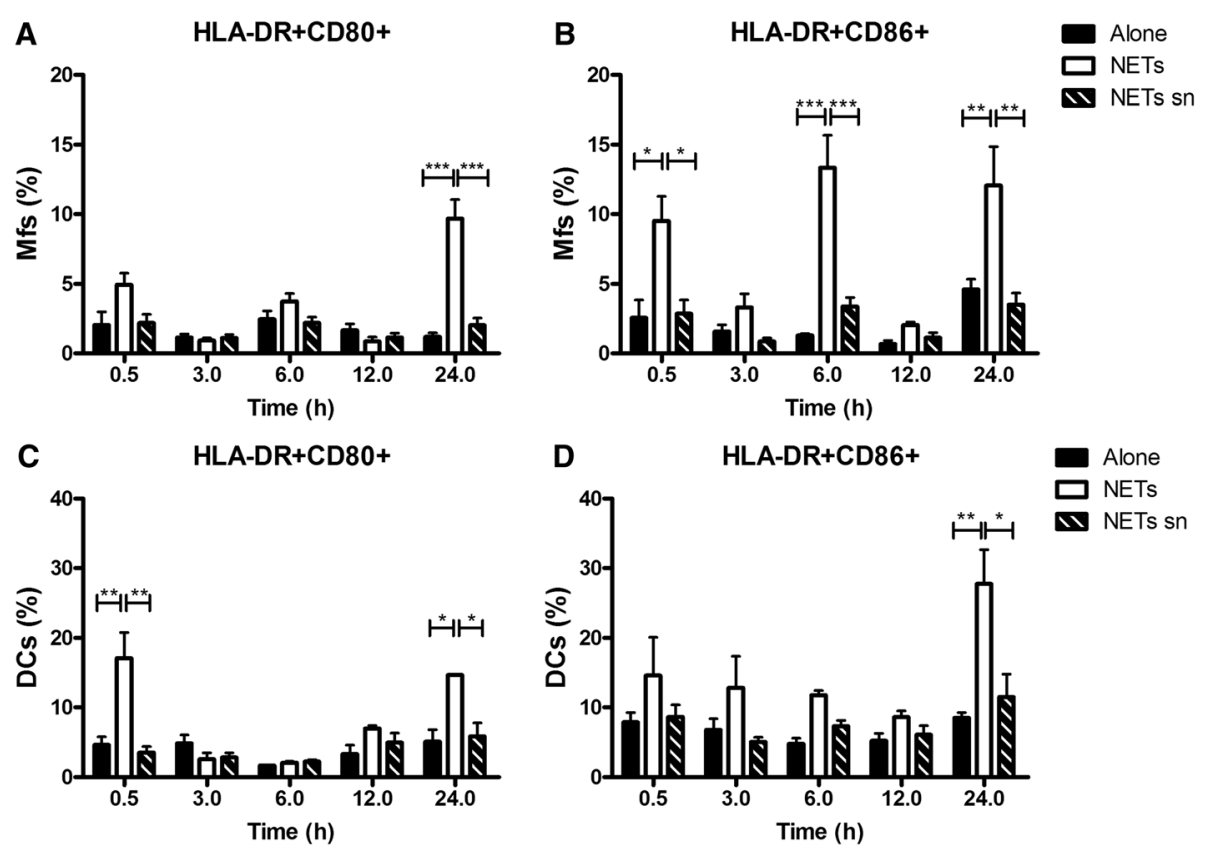

Figure 1 Activation kinetics of macrophages and dendritic cells upon exposure to NETs from human blood neutrophils. Percentage of Mfs $(\mathbf{A}, \mathbf{B})$ or DCs $(\mathbf{C}, \mathbf{D})$ labeled for HLA-DR, CD80 and CD86 upon incubation only in culture medium alone, with NETs or NETs supernatant as controls. Cells were evaluated at $0.5,3,6,12$ and 24 h post-stimulation. ${ }^{* P}=0.01$; ${ }^{* * P}=0.001$; ${ }^{* *} \mathrm{P}<0.0001$, one-way ANOVA. Mfs: Macrophages, DC: Dendritic cells, NETs sn: NETs supernatants. 

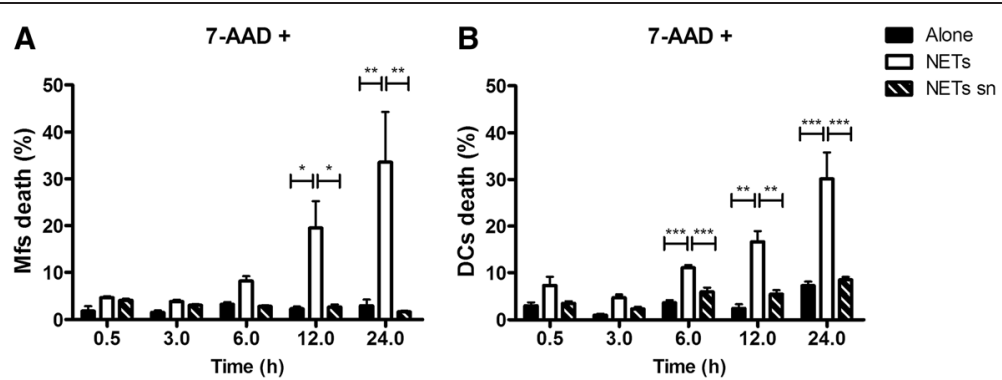

Figure 2 Death kinetics of macrophages and dendritic cells exposed to NETs from human blood neutrophils. Percentage of macrophages (A) or dendritic cells (B) stained with 7-Amino-Actinomycin-D (7AAD) after incubation with culture medium alone, with NETs or NETs supernatants. 7-AAD positive cells represent dead cells and were evaluated at $0.5,3,6,12$ and 24 h post-stimulation. ${ }^{*} \mathrm{P}=0.01$; ${ }^{* *} \mathrm{P}=0.001$; ${ }^{* *} \mathrm{P}<0.0001$, one-way ANOVA. Mfs: Macrophages, DC: Dendritic cells, NETs sn: NETs supernatants.

caspase-independent cell death. Results indicated that APCs death was not exclusively through the caspase-3 pathway because these populations (Mfs and DCs) also showed differences in AIF (Figure 4C,D). Compared to control cells we found that in macrophages exposed to NETs, AIF appeared since 30 minutes (Figure 4C). In contrast, AIF increase in DCs was seen until $12 \mathrm{~h}$ (Figure 4D).

\section{NETs caused mitochondrial morphological alterations in both APCs}

Transmission electron microscopy (TEM) has been an excellent tool in cell death research. To ascertain if NETs induced intracellular changes, we analyzed by TEM the Mfs and DCs cultured with NETs. At 30 minutes, no changes were observed in Mfs and DCs in response to NETs (Figure 5A-D). However, at 24 hours post-

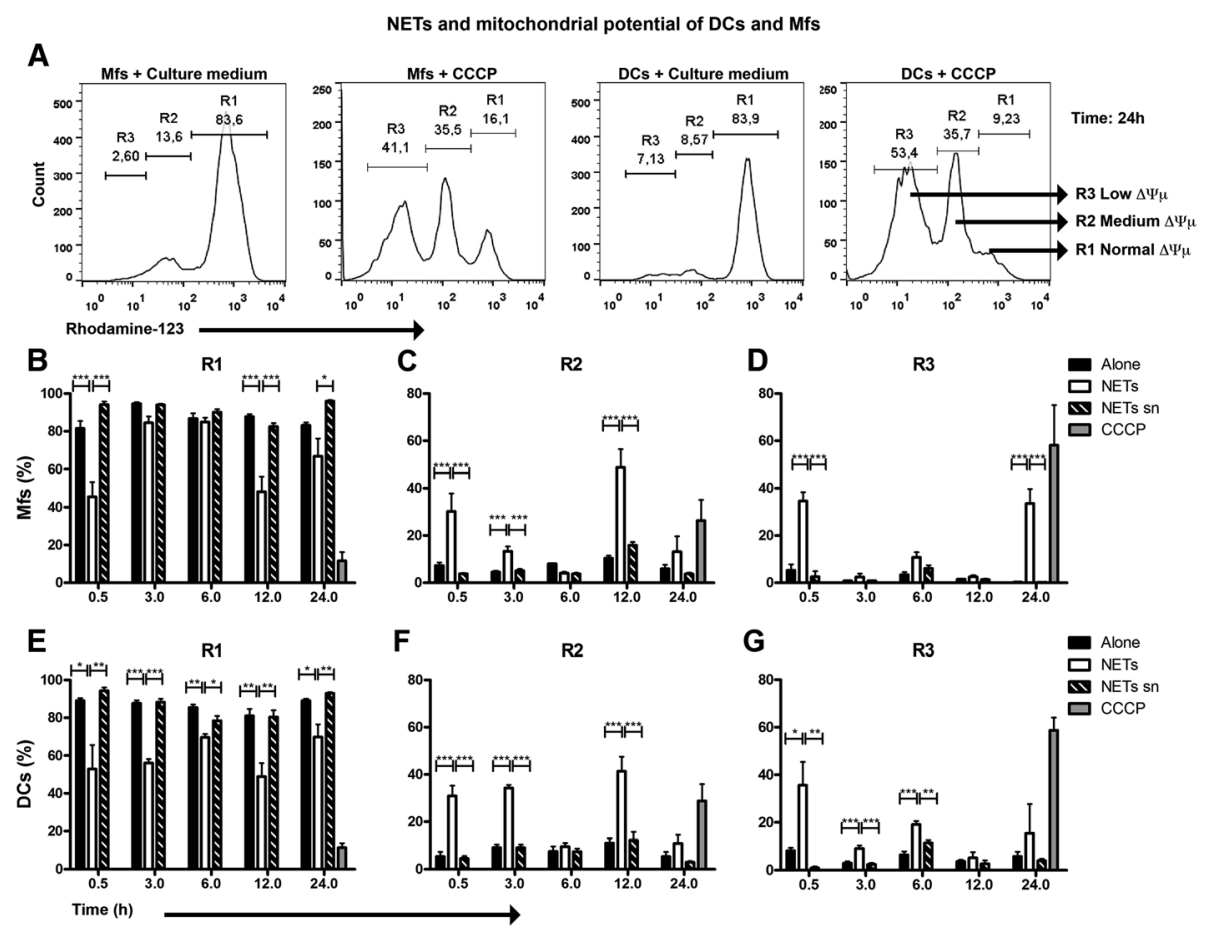

Figure 3 Analysis of mitochondrial membrane integrity from Mfs and DCs exposed to NETs or NETs supernatants. Percentage of macrophages (B-D) or dendritic cells (E-G) incubated with NETs and found in different regions of histograms upon staining with Rhodamine-123. Rhodamine-123 labeling was evaluated at 0.5, 3, 6, 12 and 24h post-incubation with NETs. The histograms shown in (A) are examples only to illustrate the regions obtained from two experimental conditions, in this example from Mfs and DCs cultured for 24 hs with medium alone or CCCP (positive control), where R1: normal $\Delta \psi_{\mu}$, R2: medium $\Delta \psi_{\mu}$, R3: low $\Delta \psi_{\mu} \Delta \psi_{\mu}$ : mitochondrial membrane potential, Mfs: Macrophages, DC: Dendritic cells, NETs sn: NETs supernatants, CCCP: Carbonyl cyanide m-chlorophenyl hydrazone. ${ }^{* P}=0.01$; ${ }^{* * P}=0.001$; ${ }^{* * * P}<0.0001$, one-way ANOVA. 

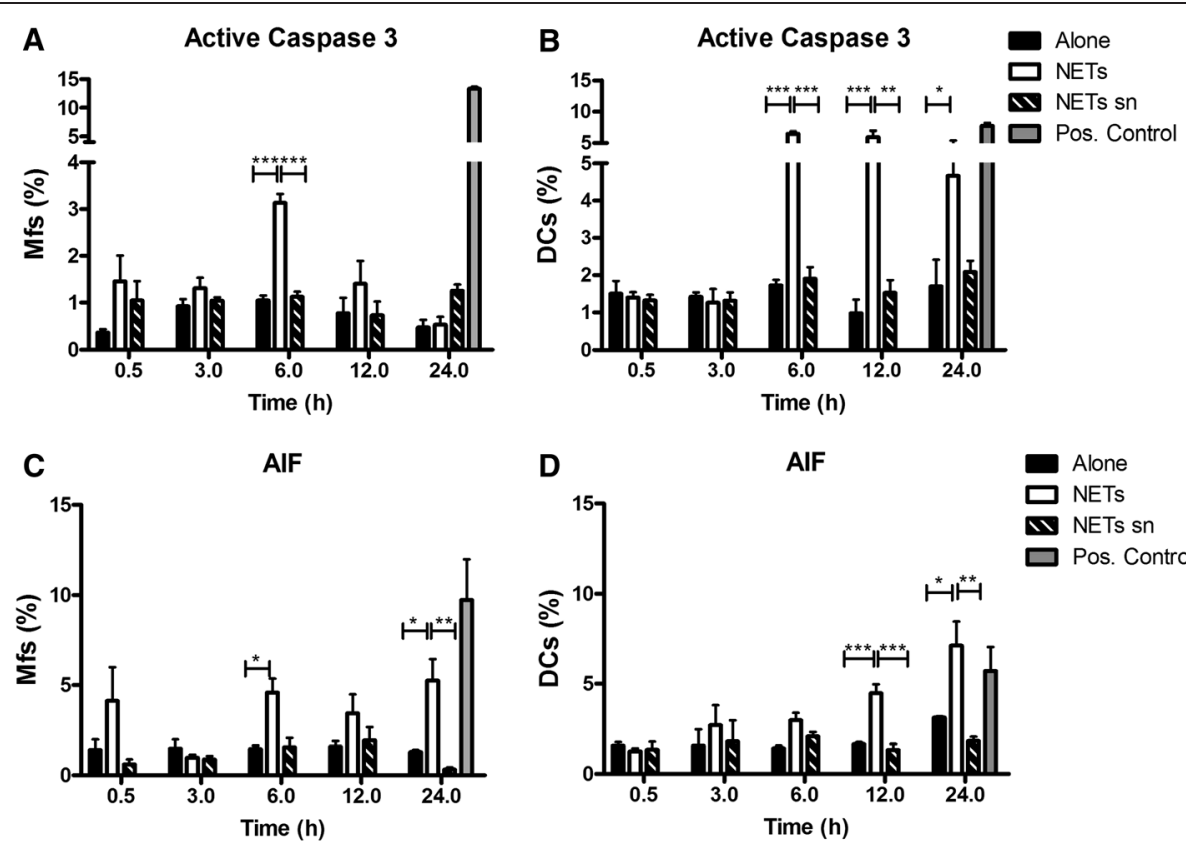

Figure 4 Kinetics of apoptosis of macrophages and dendritic cells upon exposure to NETs. Percentage of macrophages $(A, C)$ or dendritic cells (B,D) incubated only in medium, with NETs, or NETs sn, and labeled for active Caspase-3 (A,B) and AIF (C,D). Active Caspase-3 and AIF were evaluated at 0.5, 3, 6, 12 and $24 \mathrm{~h}$ post-stimulation with NETs. Positive controls represent Mfs or DCs cultured either in PBS (for Caspase-3) or with CCCP (for AIF) for 24 hours before analysis. Mfs: Macrophages, DC: Dendritic cells, NETs sn: NETs supernatants, CCCP: Carbonyl cyanide m-chlorophenyl hydrazone. ${ }^{*} \mathrm{P}=0.01{ }^{* *} \mathrm{P}=0.001 ;{ }^{* *} \mathrm{P}<0.0001$, one-way ANOVA.

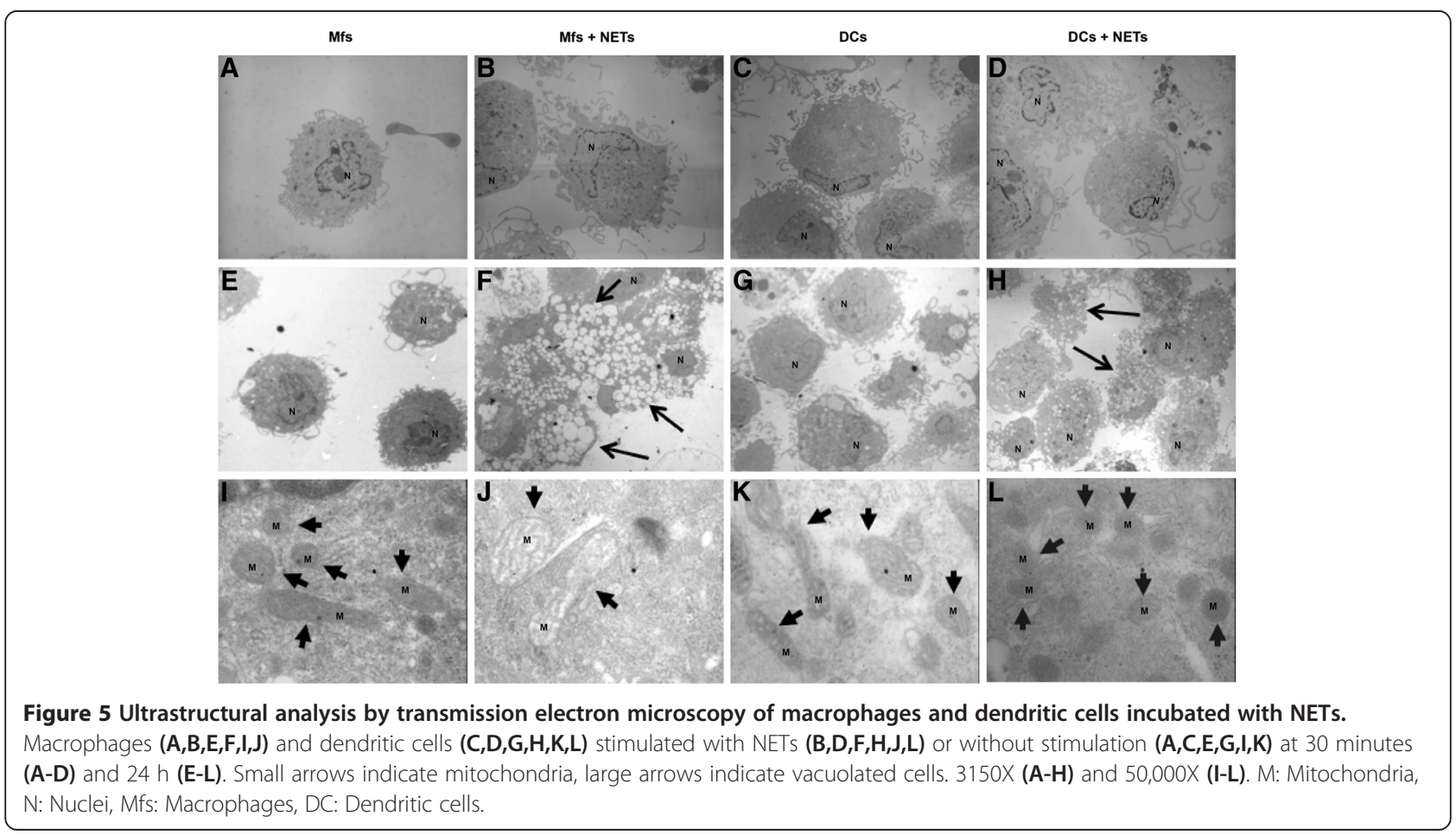


stimulation Mfs and DCs exhibited clear cytoplasmic changes (Figure 5F,H) compared with control cells (Figure 5E,G). In NET-stimulated macrophages appeared many cytoplasmic vacuoles as well as ultrastructural mitochondrial changes with loss of lamellipodia (Figure 5F) and mitochondrial crests (Figure 5J). In contrast, DCs treated with NETs had some cytoplasmic vacuoles, little loss of lamellipodia at $24 \mathrm{~h}$ post-stimulation (Figure $5 \mathrm{H}$ ) and smaller, circular mitochondria (Figure 5L).

\section{Macrophages and dendritic cells might die upon exposure to individual NETs components}

When exposing Mfs and DCs to NETs components (Histone H2A, MPO, LL-37, Elastase, Cathepsin G) for $24 \mathrm{hs}$, by means of 7AAD we observed a significant proportion of dead cells (Figure 6A,B). For Mfs this was mainly due to elastase and cathepsin $\mathrm{G}$ at concentrations of $0.1,1.0$ and $10 \mu \mathrm{g} / \mathrm{mL}$ (Figure 6A), and for DCs was at $10 \mu \mathrm{g} / \mathrm{mL}$ (Figure 6B). Although in Mfs the differences were not significant.

Using rhodamine-123 to assess the mitochondrial potential of Mfs and DCs which have been incubated with NETs components, the histograms showed two peaks: $\mathrm{R} 1$ (normal $\Delta \Psi \mathrm{m}$ ) and $\mathrm{R} 2$ (medium $\Delta \Psi \mathrm{m}$ ). Interestingly, the $\mathrm{R} 3$ region (low $\Delta \Psi \mathrm{m}$ ), the one corresponding to cells with severe damage in mitochondrial membranes, was lost when APCs were incubated only with individual NETs components (Figure 7E).

When high concentrations of histone $\mathrm{H} 2 \mathrm{~A}(10 \mu \mathrm{g} / \mathrm{ml})$ were used, the R1region (normal $\Delta \Psi \mathrm{m}$ ) decreased significantly, mainly in DCs (Figure $7 \mathrm{C}$ ). Likewise, the APCs (mainly DCs) with some damage in mitochondrial membranes, found in $\mathrm{R} 2$ region or medium $\Delta \Psi \mathrm{m}$, increased significantly when cells were incubated with histone H2A (Figure 7D),

\section{Discussion}

The leukocytes most abundant in blood are Neutrophils, which integrate the first defensive line against a great variety of microbial pathogens (bacteria, fungi, etc.). Neutrophil microbicidal activities occur through phagocytosis and degranulation (Kennedy and DeLeo 2009). The granules of human neutrophils are recognized for their high content of proteolytic and bactericidal proteins (Welsh and Spitznagel 1971).

PMNs recruited to infected tissue are able to engulf, digest and kill microorganisms as well as the debris ensuing the inflammatory process. However there are multiple components released by neutrophils that can damage neighboring tissues. Moreover professional phagocytes recognize apoptotic cells removing them, thus precluding that aged PMNs discharge their potentially toxic components (Weiss 1989).

In 2004 Brinkmann reported that when neutrophils are activated, they release to the extracellular milieu fibers composed of DNA (apparently the major structural component) and several associated molecules (histones, elastase, LL37, cathepsin G, BPI, lactoferrin, gelatinase, MPO, among others so far identified), these structures were appropriately named "neutrophil extracellular traps" (NETs) because literally trap and kill bacteria, fungi, and protozoa (von Kockritz-Blickwede and Nizet 2009). Furthermore, neutrophils elastase can degrade virulence factors from Gram-negative bacteria (Weinrauch et al. 2002). However, administering elastase into the renal artery of rats has provoked damage with massive proteinuria (Johnson et al. 1988).

While the effects of these various enzymatic components from neutrophil granules upon microbial killing have been long documented, the potential deleterious consequences upon resident and recruited cells during the inflammatory process, have been barely analyzed.

According to our data, depending on the timing of the process, NETs can induce either activation or damage to Mfs and DCs. Clayton described that human monocytesderived DCs upon ingesting human apoptotic and necrotic neutrophils, increase the expression of CD83 and MHC-II. Of note, if the ingestion of apoptotic neutrophils
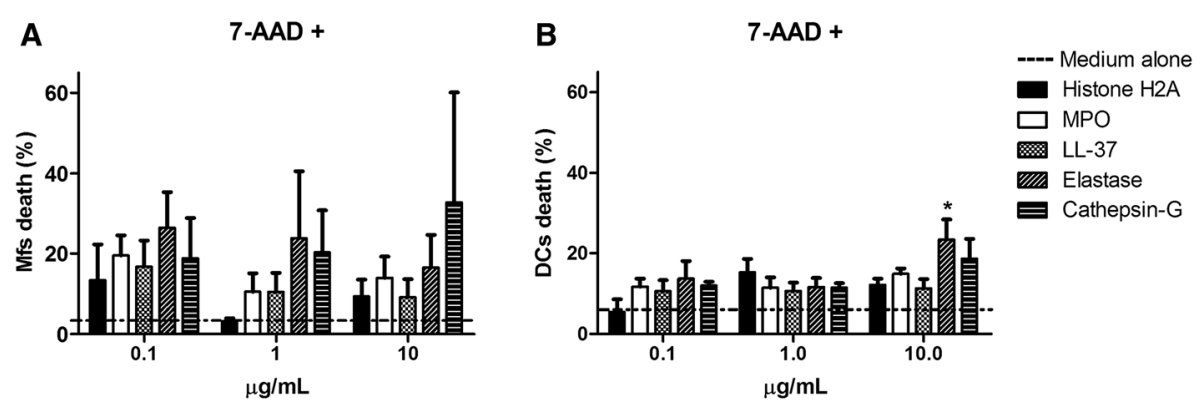

Figure 6 Effect of various concentrations of individual NETs components on the viability of Mfs and DCs. Percentage of macrophages (A) or dendritic cells (B) stained with 7-Amino-Actinomycin-D (7AAD) after incubation with Histone H2A, MPO, LL37, Elastase, Cathepsin G. 7-AAD+ cells represent dead cells and were evaluated at $24 \mathrm{~h}$ post-incubation. ${ }^{*} \mathrm{P}=0.01$. one-way ANOVA. Mfs: Macrophages, DC: Dendritic cells, MPO: Myeloperoxidase, LL-37: Cathelicidin LL-37. 

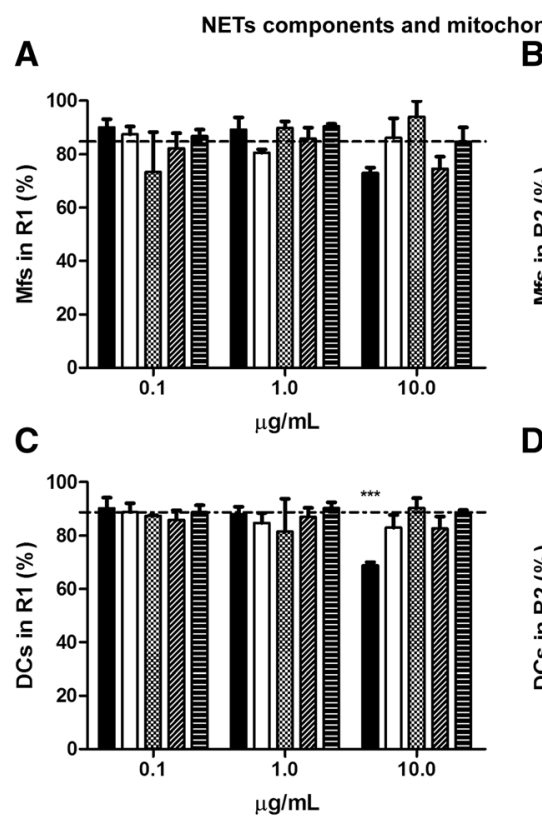

$\mathbf{E}$

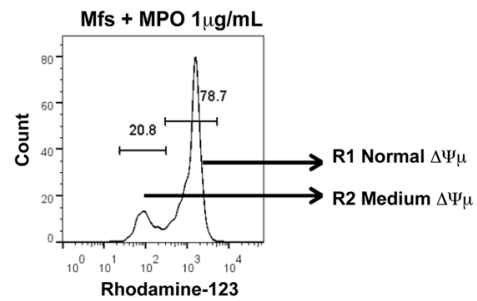

ondrial potential of Mfs and DCs

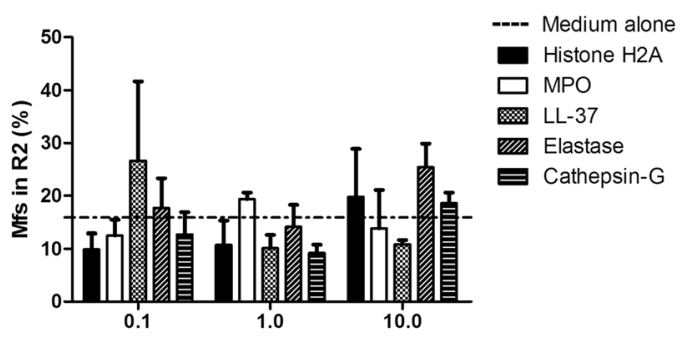

D

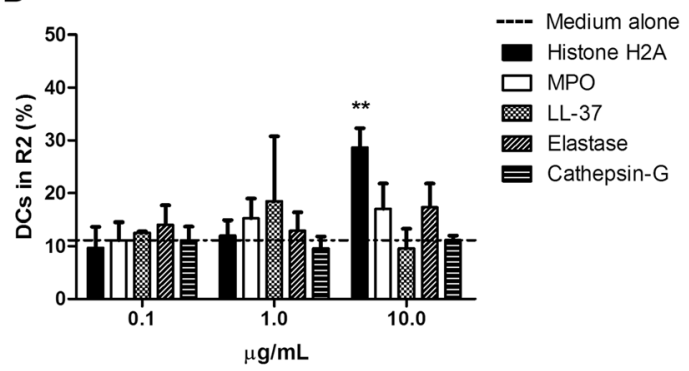

Figure 7 Analysis of mitochondrial membrane integrity from macrophages and dendritic cells exposed to individual NETs components. Percentage of macrophages (A,B) or dendritic cells (C,D) incubated with Histone H2A, MPO, LL-37, Elastase, Cathepsin G and subsequently stained with rhodamine-123. Rhodamine-123 labeling was evaluated at $24 \mathrm{~h}$ post-incubation with NETs components. Histogram in (E) is one example depicting the regions obtained from one experimental condition, in this case Mfs cultured with medium alone for $24 \mathrm{~h}$, where R1: normal $\Delta \psi \mu$, R2: medium $\Delta \psi_{\mu}$. $\Delta \psi_{\mu}$ : mitochondrial membrane potential, Mfs: Macrophages, DC: Dendritic cells, MPO: Myeloperoxidase, LL-37: Cathelicidin LL-37. ${ }^{* *} \mathrm{P}=0.001 ;{ }^{* *} \mathrm{P}<0.0001$, one-way ANOVA.

is high, it can induce down-regulation of the costimulatory molecules CD80, CD86 and CD40 (Clayton et al. 2003). Very recently it was found that NETs efficiently triggered plasmacytoid Dendritic Cell (pDC) activation (Garcia-Romo et al. 2011; Lande et al. 2011). Here we show that upon short exposure, NETs can also induce an increased expression of the costimulatory molecules CD80 and CD86 in macrophages and conventional DCs, both molecules indicative of APC activation. This APC stimulation could be important to develop efficient immune responses subsequent to the neutrophils activities.

However, it has been shown that NETs can also induce damage to neighboring cells. For instance, Gupta showed that activated endothelial cells interacting with transmigrating neutrophils were able not only of inducing NETs, but were also susceptible to NETosis-mediated cell death (Gupta et al. 2010).

We observed similar effects when NETs were added to macrophages or DCs for a relatively "prolonged" period, where the increased proportion of dead cells was noticeable at $6 \mathrm{~h}$ and clearly evident at $24 \mathrm{~h}$. When we used rhodamine-123 to assess if there was damage to the mitochondria, we found decreased mitochondrial membrane potential, suggesting an ongoing apoptotic process. This phenomenon was through the caspase- 3 and the AIF pathway. Indeed, others have shown that some components of neutrophil granules such as LL37 can induce apoptosis in $\mathrm{T}$ lymphocytes, regulatory $\mathrm{T}$ cells as well as in airways epithelial cells (Barlow et al. 2006; Mader et al. 2011a; Mader et al. 2011b). On the other hand, elastase and cathepsin G mediated glomerular injury in vivo (Johnson et al. 1988); and cathepsin G has been shown as a critical component sustaining neutrophil-mediated acute tissue pathology and fibrosis after renal ischemia/ reperfusion injury (Shimoda et al. 2007). We show here that some NETs components such as histone H2A principally, and to a lesser degree elastase, can cause mitochondrial membrane damage to Mfs and DCs if sufficient time 
is given. By electron microscopy we observed that in the cultures of APCs exposed to NETs, intracellular changes appeared at the ultrastructural level in mitochondria. This corroborated the results obtained by flow cytometry about mitochondrial membrane damage caused by NETs exposure, suggesting that it might be due to some components of NETs. However it would seem that Mfs are more prone than DCs to extended NETs exposure according to Cytochrome $\mathrm{C}$ expression (data not shown).

Our results thus suggest that interaction of NETs or certain NETs components with APCs induced first an activation process upon these cells at early times, but then -if the exposure to NETs continued- a negative effect was seen in cell survival by damage to the mitochondrial membrane, suggesting that some components of NETs might also have, later on, apoptotic effects over these APCs.

An emerging hypothetical scenario is that early in an inflammatory reaction PMNs are attracted to lesional sites, they liberate in situ the NETs with their proinflammatory content to trap and kill potential intruders, thus augmenting the initial inflammation. At this point other cells likely intervening at the inflammatory focus are the two main APCs (Mfs and DCs), which interact with the released material scavenging it while the inflammatory reaction continues. Early after this APCs-NETs interplay is started, the APCs are activated likely getting ready to induce efficient immunity. However, as this interaction continues, apoptotic death is triggered in both APCs, thus the inflammatory reaction starts to decline. As the APCs continue into apoptosis, inflammation could then decline and subside.

\section{Additional files}

Additional file 1: Figure S2. Analysis of mitochondrial membrane integrity from Mfs and DCs exposed either to PMA alone, or to NETs at two different ratios. Percentage of macrophages (A-D) or dendritic cells $(\mathbf{E}-\mathbf{H})$ incubated with medium alone $(\mathbf{A}, \mathbf{E}), 100 \mathrm{nM}$ PMA (B,F), APC 1:1 NETs $(\mathbf{C}, \mathbf{G})$ and APC 1:2 NETs $(\mathbf{D}, \mathbf{H})$ for $3 \mathrm{~h}$. Horizontal bars in the histograms indicate the region with normal $\Delta \psi_{\mu}$ (without damage). Mfs: Macrophages, DC: Dendritic cells.

Additional file 2: Figure S1. Neutrophil extracellular traps (NETs). Unstimulated (A-D) and PMA-stimulated (E-H) blood neutrophils were labeled for DNA (Blue), Elastase (Red) and Histone (Green). DNA staining (DAPI) is shown in $(\mathbf{C}, \mathbf{G})$. Immunostaining of neutrophil elastase is shown in $\mathbf{( B , F )}$, histone in $\mathbf{( A , E )}$, and the merge in $\mathbf{( D , H )}$. Stimulated PMNs were washed twice with culture medium containing 5\% FBS and the pellets were labeled for elastase, histone and DNA, as indicated, confirming the presence of NETs (E-L). Scale bars: $10 \mu \mathrm{m}$.

\section{Abbreviations}

AlF: Apoptosis-inducing factor; APCs: Antigen presenting cells; CDCs: Conventional dendritic cells; Mfs: Macrophages; NETs: Neutrophil extracelullar traps; PMNs: Polymorfonuclear leukocytes; Rh123: Rhodamine 123; $\Delta \psi_{\mu}$ : Mitochondrial membrane potential.

\section{Competing interests}

The authors declare that they have no competing interests.

\section{Authors' contributions}

LDM, LEST, IEG and LFR designed the research; LDM, MCOU, JCYP, MAGJ, YLPV and JCA performed the experiments; LDM, LEST, ACV, GSGR, MAGJ, YLPV, RCS, SEP and LFR analyzed the data; LDM, IES and LFR wrote the manuscript. All authors read and approved the final manuscript.

\section{Acknowledgements}

Authors acknowledge the help of V. Rosales, the Cytometry Unit and of Nallely A. Torres, Toxicology Department. This work was supported by SIP20110369, CONACYT Grants 105278 and 104667. Rommel Chacón-Salinas, Sergio Estrada-Parra, Luvia Sánchez-Torres, Iris Estrada-García are fellow holders of COFAA, EDI and SNI. Luis Donis-Maturano is a fellow holder from the National Council for Science and Technology Conacyt. Sergio Estrada-Parra, Luvia Sánchez-Torres, Iris Estrada-García, Juana Calderon-Amador, Arturo Cerbulo-Vazquez and Leopoldo Flores-Romo are members of the National System of Researchers of Mexico, SNI.

\section{Author details}

'Department of Cell Biology, Cinvestav-IPN. AV. IPN No 2508, Zacatenco C.P. 07330, D.F, México. ²Department of Immunology, ENCB-IPN, Col. Santo Tomas C.P. 11340D.F, Mexico. ${ }^{3}$ Department of Cell Biology, INPer., Montes Urales 800, Lomas Virreyes C.P.11000D.F, México. ${ }^{4}$ Department of Nephrology, Leiden University Medical Center, Albinusdreef 2, 2333 ZA Leiden, The Netherlands.

Received: 31 August 2014 Accepted: 17 March 2015

Published online: 02 April 2015

\section{References}

Aga E, Katschinski DM, van Zandbergen G, Laufs H, Hansen B, Muller K, Solbach W, Laskay T (2002) Inhibition of the spontaneous apoptosis of neutrophil granulocytes by the intracellular parasite Leishmania major. J Immunol 169(2):898-905

Baker VS, Imade GE, Molta NB, Tawde P, Pam SD, Obadofin MO, Sagay SA, Egah DZ, lya D, Afolabi BB, Baker M, Ford K, Ford R, Roux KH, Keller TC 3rd (2008) Cytokine-associated neutrophil extracellular traps and antinuclear antibodies in Plasmodium falciparum infected children under six years of age. Malar J 7:41, doi:10.1186/1475-2875-7-41

Barlow PG, Li Y, Wilkinson TS, Bowdish DM, Lau YE, Cosseau C, Haslett C, Simpson AJ, Hancock RE, Davidson DJ (2006) The human cationic host defense peptide LL-37 mediates contrasting effects on apoptotic pathways in different primary cells of the innate immune system. J Leukoc Biol 80(3):509-520, doi:10.1189/jlb.1005560

Beiter K, Wartha F, Albiger B, Normark S, Zychlinsky A, Henriques-Normark B (2006) An endonuclease allows Streptococcus pneumoniae to escape from neutrophil extracellular traps. Curr biol: CB 16(4):401-407, doi:10.1016/j. cub.2006.01.056

Bhatia S, Edidin M, Almo SC, Nathenson SG (2006) B7-1 and B7-2: similar costimulatory ligands with different biochemical, oligomeric and signaling properties. Immunol Lett 104(1-2):70-75, doi:10.1016/j.imlet.2005.11.019

Borregaard N, Cowland JB (1997) Granules of the human neutrophilic polymorphonuclear leukocyte. Blood 89(10):3503-3521

Brinkmann V, Reichard U, Goosmann C, Fauler B, Uhlemann Y, Weiss DS, Weinrauch Y, Zychlinsky A (2004) Neutrophil extracellular traps kill bacteria. Science 303(5663):1532-1535, doi:10.1126/science.1092385

Bruns S, Kniemeyer O, Hasenberg M, Aimanianda V, Nietzsche S, Thywissen A, Jeron A, Latge JP, Brakhage AA, Gunzer M (2010) Production of extracellular traps against Aspergillus fumigatus in vitro and in infected lung tissue is dependent on invading neutrophils and influenced by hydrophobin RodA. PLoS Pathog 6(4):e1000873, doi:10.1371/journal.ppat.1000873

Buchanan JT, Simpson AJ, Aziz RK, Liu GY, Kristian SA, Kotb M, Feramisco J, Nizet V (2006) DNase expression allows the pathogen group A Streptococcus to escape killing in neutrophil extracellular traps. Curr Biol 16(4):396-400, doi:10.1016/j. cub.2005.12.039

Caux C, Vanbervliet B, Massacrier C, Dezutter-Dambuyant C, de Saint-Vis B, Jacquet C, Yoneda K, Imamura S, Schmitt D, Banchereau J (1996) CD34+ hematopoietic progenitors from human cord blood differentiate along two independent dendritic cell pathways in response to GM-CSF + TNF alpha. J Exp Med 184(2):695-706

Clayton AR, Prue RL, Harper L, Drayson MT, Savage CO (2003) Dendritic cell uptake of human apoptotic and necrotic neutrophils inhibits CD40, CD80, 
and CD86 expression and reduces allogeneic T cell responses: relevance to systemic vasculitis. Arthritis Rheum 48(8):2362-2374, doi:10.1002/art.11130

Chen LB, Summerhayes IC, Johnson LV, Walsh ML, Bernal SD, Lampidis TJ (1982) Probing mitochondria in living cells with rhodamine 123. Cold Spring Harb Symp Quant Biol 46(Pt 1):141-155

Chuammitri P, Ostojic J, Andreasen CB, Redmond SB, Lamont SJ, Palic D (2009) Chicken heterophil extracellular traps (HETs): novel defense mechanism of chicken heterophils. Vet Immunol Immunopathol 129(1-2):126-131, doi:10.1016/j.vetimm.2008.12.013

de Graaf AO, van den Heuvel LP, Dijkman HB, de Abreu RA, Birkenkamp KU, de Witte T, van der Reijden BA, Smeitink JA, Jansen JH (2004) BCl-2 prevents loss of mitochondria in CCCP-induced apoptosis. Exp Cell Res 299(2):533-540, doi:10.1016/j.yexcr.2004.06.024

Dworski R, Simon HU, Hoskins A, Yousefi S (2011) Eosinophil and neutrophil extracellular DNA traps in human allergic asthmatic airways. J Allergy Clin Immunol 127(5):1260-1266, doi:10.1016/j.jaci.2010.12.1103

Ermert D, Urban CF, Laube B, Goosmann C, Zychlinsky A, Brinkmann V (2009) Mouse neutrophil extracellular traps in microbial infections. J Innate Immun 1(3):181-193, doi:10.1159/000205281

Fuchs TA, Abed U, Goosmann C, Hurwitz R, Schulze I, Wahn V, Weinrauch Y, Brinkmann V, Zychlinsky A (2007) Novel cell death program leads to neutrophil extracellular traps. J Cell Biol 176(2):231-241, doi:10.1083/ jcb.200606027

Fujie K, Shinguh Y, Inamura N, Yasumitsu R, Okamoto M, Okuhara M (1999) Release of neutrophil elastase and its role in tissue injury in acute inflammation: effect of the elastase inhibitor, FR134043. Eur J Pharmacol 374(1):117-125

Gabriel C, McMaster WR, Girard D, Descoteaux A (2010) Leishmania donovani promastigotes evade the antimicrobial activity of neutrophil extracellular traps. J Immunol 185(7):4319-4327, doi:10.4049/jimmunol.1000893

Garcia-Romo GS, Caielli S, Vega B, Connolly J, Allantaz F, Xu Z, Punaro M, Baisch J, Guiducci C, Coffman RL, Barrat FJ, Banchereau J, Pascual V (2011) Netting neutrophils are major inducers of type I IFN production in pediatric systemic lupus erythematosus. Sci Trans Med 3(73):73ra20, doi:10.1126/scitransImed.3001201

Gupta AK, Joshi MB, Philippova M, Erne P, Hasler P, Hahn S, Resink TJ (2010) Activated endothelial cells induce neutrophil extracellular traps and are susceptible to NETosis-mediated cell death. FEBS Lett 584(14):3193-3197, doi:10.1016/j.febslet.2010.06.006

Johnson LV, Walsh ML, Chen LB (1980) Localization of mitochondria in living cells with rhodamine 123. Proc Natl Acad Sci U S A 77(2):990-994

Johnson RJ, Couser WG, Alpers CE, Vissers M, Schulze M, Klebanoff SJ (1988) The human neutrophil serine proteinases, elastase and cathepsin $\mathrm{G}$, can mediate glomerular injury in vivo. J Exp Med 168(3):1169-1174

Kennedy AD, DeLeo FR (2009) Neutrophil apoptosis and the resolution of infection. Immunol Res 43(1-3):25-61, doi:10.1007/s12026-008-8049-6

Kessenbrock K, Krumbholz M, Schonermarck U, Back W, Gross WL, Werb Z, Grone HJ, Brinkmann V, Jenne DE (2009) Netting neutrophils in autoimmune small-vessel vasculitis. Nat Med 15(6):623-625, doi:10.1038/nm.1959

Lande R, Ganguly D, Facchinetti V, Frasca L, Conrad C, Gregorio J, Meller S, Chamilos G, Sebasigari R, Riccieri V, Bassett R, Amuro H, Fukuhara S, Ito T, Liu YJ, Gilliet M (2011) Neutrophils activate plasmacytoid dendritic cells by releasing self-DNA-peptide complexes in systemic lupus erythematosus. Sci Transl Med 3(73):73ra19, doi:10.1126/scitranslmed.3001180

Lim ML, Minamikawa T, Nagley P (2001) The protonophore CCCP induces mitochondrial permeability transition without cytochrome $c$ release in human osteosarcoma cells. FEBS Lett 503(1):69-74

Lippolis JD, Reinhardt TA, Goff JP, Horst RL (2006) Neutrophil extracellular trap formation by bovine neutrophils is not inhibited by milk. Vet Immunol Immunopathol 113(1-2):248-255, doi:10.1016/j.vetimm.2006.05.004

Mader JS, Ewen C, Hancock RE, Bleackley RC (2011a) The human cathelicidin, LL-37, induces granzyme-mediated apoptosis in regulatory T cells. J Immunother 34(3):229-235, doi:10.1097/CJl.0b013e318207ecdf

Mader JS, Marcet-Palacios M, Hancock RE, Bleackley RC (2011b) The human cathelicidin, LL-37, induces granzyme-mediated apoptosis in cytotoxic T lymphocytes. Exp Cell Res 317(4):531-538, doi:10.1016/j.yexcr.2010.11.015

O'Brien MC, Bolton WE (1995) Comparison of cell viability probes compatible with fixation and permeabilization for combined surface and intracellular staining in flow cytometry. Cytometry 19(3):243-255, doi:10.1002/cyto.990190308

Palic D, Andreasen CB, Ostojic J, Tell RM, Roth JA (2007) Zebrafish (Danio rerio) whole kidney assays to measure neutrophil extracellular trap release and degranulation of primary granules. J Immunol Methods 319(1-2):87-97, doi:10.1016/j.jim.2006.11.003
Papayannopoulos V, Zychlinsky A (2009) NETs: a new strategy for using old weapons. Trends Immunol 30(11):513-521, doi:10.1016/j.it.2009.07.011

Ramos-Kichik V, Mondragon-Flores R, Mondragon-Castelan M, Gonzalez-Pozos S, Muniz-Hernandez S, Rojas-Espinosa O, Chacon-Salinas R, Estrada-Parra S, Estrada-Garcia I (2009) Neutrophil extracellular traps are induced by Mycobacterium tuberculosis. Tuberculosis 89(1):29-37, doi:10.1016/j. tube.2008.09.009

Savchenko AS, Inoue A, Ohashi R, Jiang S, Hasegawa G, Tanaka T, Hamakubo T, Kodama T, Aoyagi Y, Ushiki T, Naito M (2011) Long pentraxin 3 (PTX3) expression and release by neutrophils in vitro and in ulcerative colitis. Pathol Int 61(5):290-297, doi:10.1111/j.1440-1827.2011.02651.x

Schmid I, Krall WJ, Uittenbogaart CH, Braun J, Giorgi JV (1992) Dead cell discrimination with 7-amino-actinomycin D in combination with dual color immunofluorescence in single laser flow cytometry. Cytometry 13(2):204-208, doi:10.1002/cyto.990130216

Schmid I, Uittenbogaart CH, Giorgi JV (1994) Sensitive method for measuring apoptosis and cell surface phenotype in human thymocytes by flow cytometry. Cytometry 15(1):12-20, doi:10.1002/cyto.990150104

Segal AW (2005) How neutrophils kill microbes. Annu Rev Immunol 23:197-223, doi:10.1146/annurev.immunol.23.021704.115653

Shimoda N, Fukazawa N, Nonomura K, Fairchild RL (2007) Cathepsin g is required for sustained inflammation and tissue injury after reperfusion of ischemic kidneys. Am J Pathol 170(3):930-940, doi:10.2353/ajpath.2007.060486

Vitkov L, Klappacher M, Hannig M, Krautgartner WD (2009) Extracellular neutrophil traps in periodontitis. J Periodontal Res 44(5):664-672, doi:10.1111/j.1600-0765.2008.01175.x

von Kockritz-Blickwede M, Nizet V (2009) Innate immunity turned inside-out: antimicrobial defense by phagocyte extracellular traps. J Mol Med 87(8):775-783, doi:10.1007/s00109-009-0481-0

Wardini AB, Guimaraes-Costa AB, Nascimento MT, Nadaes NR, Danelli MG, Mazur C, Benjamim CF, Saraiva EM, Pinto-da-Silva LH (2010) Characterization of neutrophil extracellular traps in cats naturally infected with feline leukemia virus. J Gen Virol 91(Pt 1):259-264, doi:10.1099/vir. 0.014613-0

Weinrauch Y, Drujan D, Shapiro SD, Weiss J, Zychlinsky A (2002) Neutrophil elastase targets virulence factors of enterobacteria. Nature 417(6884):91-94, doi:10.1038/417091a

Weiss SJ (1989) Tissue destruction by neutrophils. N Engl J Med 320(6):365-376, doi:10.1056/NEJM198902093200606

Welsh IR, Spitznagel JK (1971) Distribution of lysosomal enzymes, cationic proteins, and bactericidal substances in subcellular fractions of human polymorphonuclear leukocytes. Infect Immun 4(2):97-102

\section{Submit your manuscript to a SpringerOpen ${ }^{\odot}$ journal and benefit from:}

- Convenient online submission

- Rigorous peer review

- Immediate publication on acceptance

- Open access: articles freely available online

- High visibility within the field

- Retaining the copyright to your article

Submit your next manuscript at springeropen.com 